\title{
Targeting miRNAs in Osteoblast Differentiation under Malnutrition Conditions
}

\author{
Yuying Wang1, Rui He², Liangjun Zhong² \\ ${ }^{1}$ College of Life and Environmental Science, Hangzhou Normal University, Hangzhou, China \\ ${ }^{2}$ College of Medicine, Hangzhou Normal University, Hangzhou, China \\ Email: yywwhz@163.com
}

How to cite this paper: Wang, Y.Y., He, R. and Zhong, L.J. (2018) Targeting miRNAs in Osteoblast Differentiation under Malnutrition Conditions. Journal of Biosciences and Medicines, 6, 111-119.

https://doi.org/10.4236/jbm.2018.65012

Received: March 20, 2018

Accepted: May 21, 2018

Published: May 24, 2018

Copyright ( 92018 by authors and Scientific Research Publishing Inc. This work is licensed under the Creative Commons Attribution International License (CC BY 4.0).

http://creativecommons.org/licenses/by/4.0/

\section{cc) (i) Open Access}

\begin{abstract}
Aims: Previous studies reported that reduced bone formation was identified in fasting adult female mice compared with the ad libitum control group. An increasing number of studies have shown that miRNAs contribute to bone homeostasis. Unfortunately, there are minor concerns about the underlying mechanisms in osteoblastic differentiation under malnutrition conditions. Methods: We investigated microRNAs (miRNAs) in osteoblastic differentiation under malnutrition conditions using high-throughput bioinformatics approaches. To screen for targeted microRNAs, sequences were quantified by aligning reads to miRbase using miRDeep2 software. Unadjusted p-values were calculated using the Student's t-test. Genes with a p-value of $<0.05$ and $\log 2 \mathrm{FC}$ (fold change) $\geq 1$ were considered differentially expressed genes (DEGs). DEGs were submitted to Gene ontology (GO) and Kyoto Encyclopedia of Genes and Genomes (KEGG) enrichment analyses, respectively. Results: They were mainly enriched in biological process terms type and biological pathways, respectively. Particularly, we evaluated seven microRNAs, mir-494 3p, mir-466, mir-455, mir-708, mir-298, mir-92 and mir-224, which likely play roles in osteoblastogenesis in fasting adult mice. Conclusion: To our knowledge, this is the first study on the expression pattern of miRNA in osteoblasts of malnourished adult mice. These targeting miRNAs may provide a potential therapeutic approach to treat osteoporosis.
\end{abstract}

\section{Keywords}

RNA-Seq, microRNA, Osteoblast, Bone Homeostasis

\section{Introduction}

The maintenance of total bone mass under normal conditions is a balance of os- 
teogenesis by osteoblasts and resorption by osteoclasts. The loss of bone mass under pathological conditions is primarily caused by the failure of bone turnover, which includes decreased bone formation and enhanced bone resorption. Energy intake influences bone turnover in anorexia nervosa patients, which are commonly young exercising women or elderly underweight women [1] [2] [3]. Reduced bone formation has been observed in dietary restriction mice [4] [5]. Interestingly, bone formation and non-bone resorption were significantly changed in adult female mice between the ad libitum and the fasting groups [6].

The osteoblast lineage arises from mesenchymal stromal cells, which are regulated by genetic and epigenetic mechanisms. Emerging evidence indicates that microRNAs (miRNAs) play crucial roles in this process, from embryonic skeletal development to the maintenance of adult bone tissue [7] [8] [9]. The small non-coding RNAs, miRNAs, post-transcriptionally regulate gene expression. Unfortunately, there is no information about the regulatory mechanisms of miRNAs in osteoblastic differentiation under malnutrition conditions. To gain further insight into these molecular mechanisms, we carried out miRNA-seq using osteoblast-enriched cells derived from lower limbs. Differentially expressed genes (DEGs) in the AL and FA groups were screened. Subsequently, the underlying functions of these two sets of DEGs were investigated by gene ontology (GO) function and Kyoto Encyclopedia of Genes and Genomes (KEGG) pathway enrichment analyses. In total, we identified 103 DEGs, including 68 upand 35 down-regulated genes. Among them, 6 had high potential for functioning in osteoblastogenesis based on previous studies. Here, we report that these miRNAs may play important roles in bone formation.

\section{Materials and Methods}

\subsection{Animals}

Before the study, all experiments were reviewed and approved by the Animal Care and Use Committee of Hangzhou Normal University, Zhejiang, China. Twelve week-old C57BL/6J female mice were housed in the Hangzhou Normal University Animal Center. Mice were maintained on a 12/12-hour light/dark cycle under controlled temperature and humidity conditions. Mice were randomly divided into two groups ( $n=6 /$ group): the food ad libitum control group and the experimental group that underwent complete fasting with water ad libitum. Fasting duration (3 days) was chosen based on the experiment conducted in previous researches [4] [10]. At the start of the study, mice in the fasting group were transferred to a clean cage without access to food at Zeitgeber time 20 (ZT20). These mice were subsequently sacrificed at ZT8 on the third day of fasting [10].

\subsection{High-Throughput microRNA Sequencing}

To separate osteoblasts from the whole bone, muscle, connective tissue and periosteum were removed from femurs. Bone marrow cells in the diaphyses of fe- 
murs, which were cut at the metaphyses, were flushed out using phosphate-buffered saline. Osteoblast-enriched cells were collected using a micro-interdental brush (Kobayashi Pharmaceutical Co. Ltd., Osaka, Japan).

Total RNA was extracted using a Qiagen miRNeasy Mini Kit. The integrity of RNA was verified by running it on a $1 \%$ agarose gel and staining with ethidium bromide. The concentration of RNA was measured using a Nanodrop 2000 (Thermo, USA). Purified RNAs were used to construct small RNA libraries according to Illumina's recommendations. Briefly, purified sRNAs were ligated with 3' and 5' adapters (Illumina, San Diego, CA, USA). The first strand of cDNA was synthesised using reverse transcription, and the synthesised cDNAs were subjected to a PCR amplification process of 15 cycles, each consisting of the basic steps of denaturation $\left(10 \mathrm{~s}\right.$ at $\left.98^{\circ} \mathrm{C}\right)$, annealing $\left(30 \mathrm{~s}\right.$ at $\left.60^{\circ} \mathrm{C}\right)$ and extension $\left(15 \mathrm{~s}\right.$ at $72^{\circ} \mathrm{C}$ ). The amplified products (approximately $140 \mathrm{bp}$ ) were purified by $6 \%$ polyacrylamide gel electrophoresis. Following the purification of amplified cDNAs, products were sequenced on a Hiseq2000 (Illumina, San Diego, CA, USA) using the Illumina paired-end/single-end RNA-seq approach.

\subsection{Screening and Functional annotation for DEGs}

The sequences were quantified by aligning reads to miRbase (version 21, http://www.mirbase.org) using the miRDeep2 package [11]. The expression of miRNAs was normalised by transcripts per million (read count ${ }^{*} 10^{6} /$ total transcript count). Analysis of the differential expression of miRNA was performed using Bioconductor's package DEGs. P-values were estimated using a negative binomial distribution model and local regression to estimate the relationship between the dispersion and the mean of each miRNA. Raw p-values were considered statistically significant for $\mathrm{p}<0.05$, were adjusted for multiple testing using the Benjamini and Hochberg procedure with a $10 \%$ false discovery rate threshold. A fold change (FC) threshold of 2 (or $\log 2 \mathrm{FC}>1$ ) was used to select modulated miRNAs.

Functional enrichment of target genes was assessed based on the biological process category in GO [12] and KEGG annotation terms [13]. GO and KEGG signalling pathway analyses were performed using the GO Function package (version 1.14.0) in Bioconductor

(http://www.bioconductor.org/packages/release/bioc/html/GOFunction.html)

[14], which conducted the standard hypergeometric test. A p-value of $<0.05$ was considered to indicate a statistically significant difference.

\section{Results}

\subsection{Screening for DEGs}

Between samples from the fasting and control groups, we identified 103 DEGs with a p-value $<0.05$ and $\log 2 \mathrm{FC} \geq 1$, including 68 up- and 35 down-regulated genes. Some microRNAs identified were clearly osteoblastogenesis-related genes [15]-[21]. Among others, seven were likely to have a role in the process of os- 
teoblastogenesis. We show detailed information on them in Table 1.

\subsection{Functional and Pathway Analyses of DEGs}

According to the GO and KEGG databases, we analysed the functions and pathways of all the predicted target genes from differentially expressed miRNAs. Significant functions and pathways were filtered based on p-values $<0.05$. These genes were classified into functional categories based on GO predictions for biological processes and molecular function. As shown in Figure 1, the main GO categories for these genes were biological regulation, binding, single-organism cellular process, regulation of biological process, cellular response to stimulus and metabolic processes. In addition, target genes were enriched in the MAPK signalling pathway, axon guidance, the Wnt signalling pathway, focal adhesion, endocytosis, the $\mathrm{T}$ cell receptor signalling pathway and pathways involved in cancer (Figure 2).

\section{Discussions}

A class of short noncoding single stranded RNAs, miRNAs, have emerged as essential posttranscriptional regulators of gene expression. An increasing number of studies have shown that miRNAs contribute to bone homeostasis, which suggests that targeting miRNAs may provide a therapeutic approach to treat

Table 1. Typical DEGs screened between the AL and FA groups.

\begin{tabular}{|c|c|c|c|}
\hline microRNA ID & $\log 2 \mathrm{FC}$ & $P$ value & Description in previous studies \\
\hline \multicolumn{4}{|c|}{ Up-regulated genes } \\
\hline $\operatorname{mir}-4943 p$ & 3.5153 & $7.64 \mathrm{E}-11$ & $\begin{array}{l}\text { Inhibits proliferation of MC3T3E1 cells [22]; inhibits prolif- } \\
\text { eration and metastasis of osteosarcoma [23]; induces cellular } \\
\text { senescence of oral squamous carcinoma cells [24]. }\end{array}$ \\
\hline $\operatorname{mir}-466$ & 2.9299 & $1.08 \mathrm{E}-10$ & $\begin{array}{l}\text { Inhibits tumour growth and bone metastasis in prostate } \\
\text { cancer [25]; inhibits lymph angiogenesis [26] }\end{array}$ \\
\hline $\operatorname{mir}-455$ & 2.4634 & $3.25 \mathrm{E}-23$ & $\begin{array}{l}\text { Shows increased expression in the course of chondrogenesis } \\
\text { and OA cartilage [27]; a tumour suppressor [28]. }\end{array}$ \\
\hline $\operatorname{mir}-708$ & 1.6471 & 0.0006 & $\begin{array}{l}\text { Promotes steroid-induced osteonecrosis of femoral head and } \\
\text { suppresses osteogenic differentiation [29] }\end{array}$ \\
\hline \multicolumn{4}{|c|}{ Down-regulated genes } \\
\hline $\operatorname{mir}-298$ & 2.7127 & $2.85 \mathrm{E}-08$ & $\begin{array}{l}\text { Counteracts mutant androgen receptor toxicity in spinal and } \\
\text { bulbar muscular atrophy [30]; raises the propensity to apop- } \\
\text { tosis of aTC1-6 cells [31]. }\end{array}$ \\
\hline $\operatorname{mir}-92$ & 1.5842 & $8.56 \mathrm{E}-06$ & $\begin{array}{l}\text { promotes growth and metastasis of cancer cells [32]; deletion } \\
\text { of miR-92a is sufficient to induce a developmental skeletal } \\
\text { defect [33]; has potential to be a biomarker of osteogenesis } \\
\text { imperfecta [34]; }\end{array}$ \\
\hline $\operatorname{mir}-224$ & 1.1107 & $5.23 \mathrm{E}-06$ & $\begin{array}{l}\text { Suppresses osteosarcoma cell proliferation, migration and } \\
\text { invasion [35]; promotes the metastasis of breast cancer cells } \\
{[36] \text {. }}\end{array}$ \\
\hline
\end{tabular}




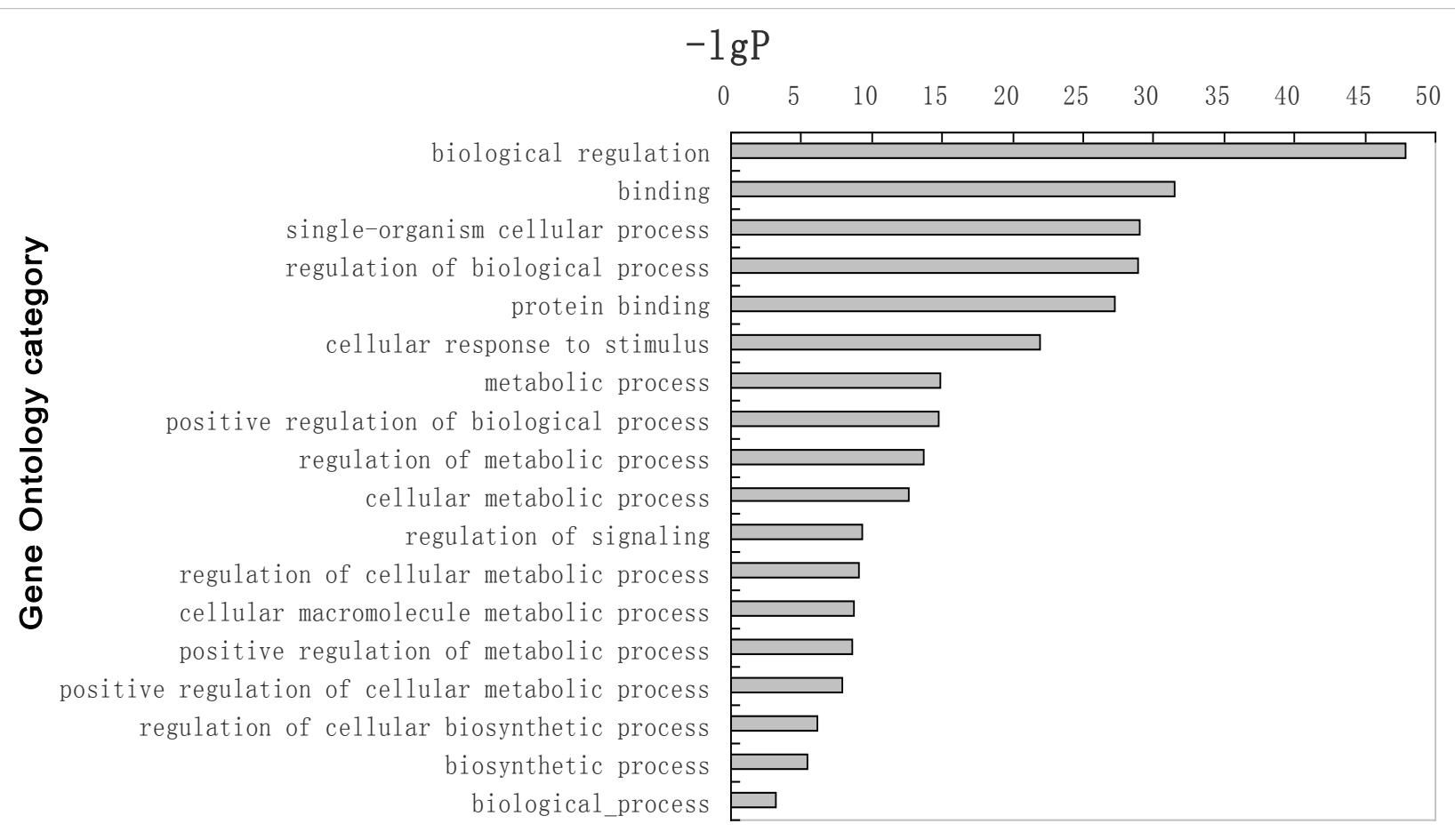

GO analysis for putative target genes. A p-value of $<0.05$ and a false discovery rate of $<0.05$ were used as thresholds to select significant GO categories; $\lg \mathrm{P}$ is the negative logarithm of the $\mathrm{p}$-value.

Figure 1. GO analysis for differentially expressed genes (DEGs).

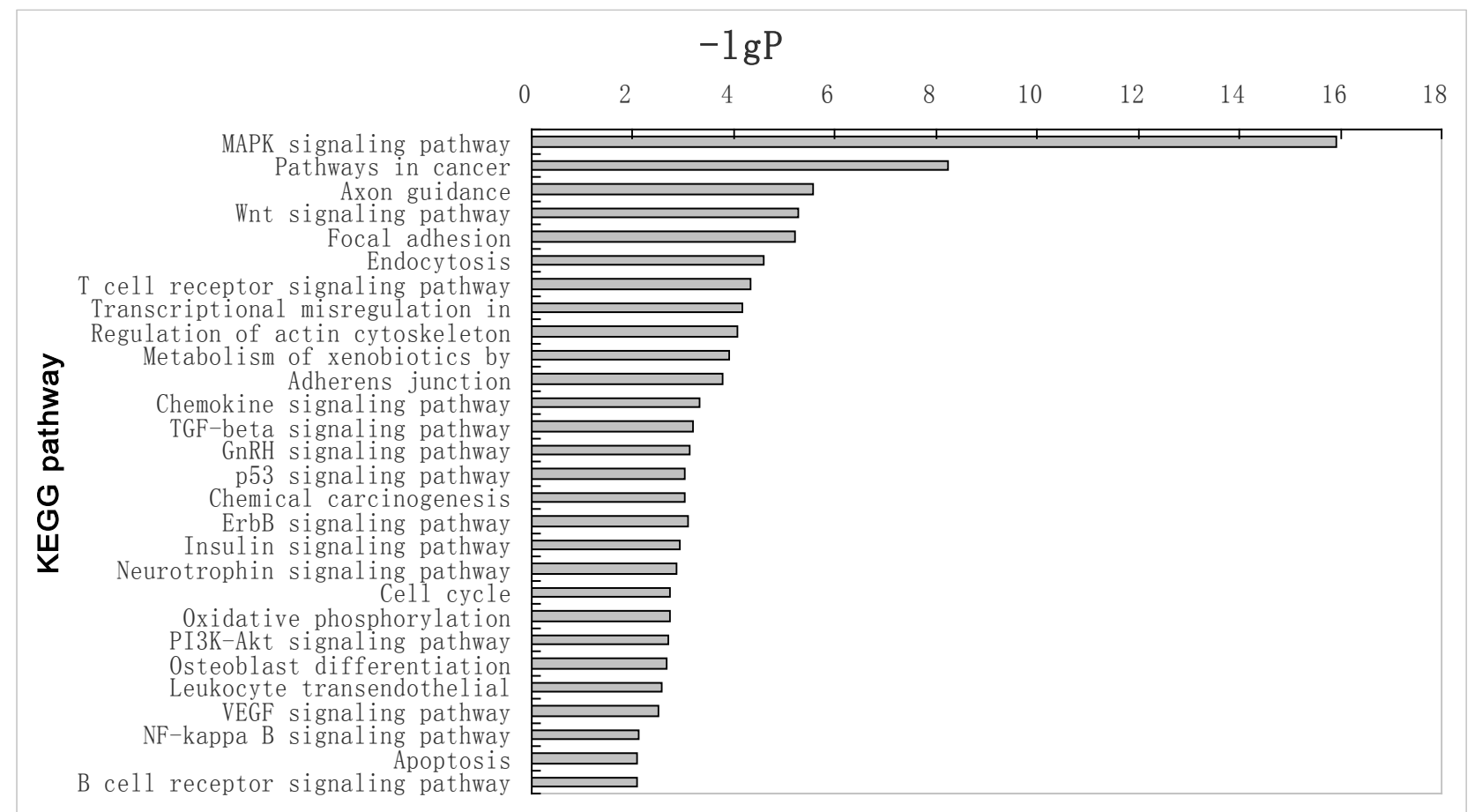

KEGG pathway analysis for putative target genes. A p-value of $<0.05$ and a false discovery rate of $<0.05$ were used as the threshold to select significant KEGG pathways; $\lg \mathrm{P}$ is the negative logarithm of the $\mathrm{p}$-value.

Figure 2. KEGG pathway analysis for differentially expressed genes (DEGs). 
osteoporosis [37]. Unfortunately, there are minor concerns about the underlying mechanisms in osteoblastic differentiation under malnutrition conditions. We investigated miRNAs identified in osteoblast-enriched cells analysed using high-throughput bioinformatics approaches.

The DEGs include genes that were clearly or potentially related to osteoblastogenesis. To evaluate their functions, GO and KEGG analyses were performed for DEGs. They were mainly enriched in biological process terms type. Additionally, target microRNAs, especially the seven microRNAs potentially involved in osteoblastogenesis, were enriched in the MAPK signalling pathway (mir-298, mir-224), Wnt signalling pathway (mir-224), TGF-beta signalling pathway (mir-455, mir-708) and Akt signalling pathway (mir-494 3p, mir-466, mir-92).

To our knowledge, this is the first study to examine miRNA expression patterns in osteoblasts of adult mice under malnutrition conditions. However, the detailed functions associated with these DEGs in osteoblastic differentiation require further investigation, and the target genes of these miRNAs need experimental validation.

\section{Acknowledgements}

This work was supported by National Natural Science Foundation of China (Grant number: 81401811).

\section{References}

[1] Fazeli, P.K. and Klibanski, A. (2014) Anorexia Nervosa and Bone Metabolism. Bone, 66, 39-45.

[2] Ihle, R. and Loucks, A.B. (2004) Dose-Response Relationships between Energy Availability and Bone Turnover in Young Exercising Women. Journal of Bone and Mineral Research, 19, 1231-1240. https://doi.org/10.1359/JBMR.040410

[3] Devine, A., Dick, I.M., Islam, A.F., Dhaliwal, S.S. and Prince, R.L. (2005) Protein Consumption Is an Important Predictor of Lower Limb Bone Mass in Elderly Women. The American Journal of Clinical Nutrition, 81, 1423-1428. https://doi.org/10.1093/ajcn/81.6.1423

[4] Brochmann, E.J., Duarte, M.E., Zaidi, H.A. and Murray, S.S. (2003) Effects of Dietary Restriction on Total Body, Femoral, and Vertebral Bone in SENCAR, C57BL/6, and DBA/2 Mice. Metabolism, 52, 1265-1273. https://doi.org/10.1016/S0026-0495(03)00194-X

[5] Shushimita, S., de Bruijn, M.J., de Bruin, R.W., IJzermans, J.N., Hendriks, R.W. and Dor, F.J. (2014) Dietary Restriction and Fasting Arrest B and T Cell Development and Increase Mature B and T Cell Numbers in Bone Marrow. PLoS ONE, 9, e87772. https://doi.org/10.1371/journal.pone.0087772

[6] Chen, J.J., He, R., Li, J., Zhang, Y., Qi, J.W., Meng, X.H., et al. (2016) Severe Impaired Bone Formation Was Induced by Short-Term Fasting in Adult Mice. American Journal of Clinical and Experimental Medicine, 4, 212-215. https://doi.org/10.11648/j.ajcem.20160406.19

[7] Hu, R., Li, H., Liu, W., Yang, L., Tan, Y.F. and Luo, X.H. (2010) Targeting miRNAs in Osteoblast Differentiation and Bone Formation. Expert Opinion on Therapeutic Targets, 14, 1109-1120. https://doi.org/10.1517/14728222.2010.512916 
[8] Taipaleenmäki, H., Bjerre Hokland, L., Chen, L., Kauppinen, S. and Kassem, M. (2012) Micro-RNAs: Targets for Enhancing Osteoblast Differentiation and Bone Formation. European Journal of Endocrinology, 166, 359-371. https://doi.org/10.1530/EJE-11-0646

[9] Lian, J.B., Stein, G.S., van Wijnen, A.J., Stein, J.L., Hassan, M.Q., Gaur, T., et al. (2012) MicroRNA Control of Bone Formation and Homeostasis. Nature Reviews Endocrinology, 31, 212-227. https://doi.org/10.1038/nrendo.2011.234

[10] Talbott, S.M. and Shapses, S.A. (1998) Fasting and Energy Intake Influence Bone Turnover in Lightweight Male Rowers. International Journal of Sport Nutrition, 8 , 377-387. https://doi.org/10.1123/ijsn.8.4.377

[11] Friedländer, M.R., Mackowiak, S.D., Li, N., Chen, W. and Rajewsky, N. (2012) miRDeep2 Accurately Identifies Known and Hundreds of Novel MicroRNA Genes in Seven Animal Clades. Nucleic Acids Research, 40, 37-52. https://doi.org/10.1093/nar/gkr688

[12] Ashburner, M., Ball, C.A., Blake, J.A., Botstein, D., Butler, H., Cherry, J.M., et al. (2000) Gene Ontology: Tool for the Unification of Biology. The Gene Ontology Consortium. Nature Genetics, 25, 25-29. https://doi.org/10.1038/75556

[13] Kanehisa, M. and Goto, S. (2000) KEGG: Kyoto Encyclopedia of Genes and Genomes. Nucleic Acids Research, 28, 27-30. https://doi.org/10.1093/nar/28.1.27

[14] Wang, J., Zhou, X., Zhu, J., Gu, Y., Zhao, W., Zou, J., et al. (2012) GO-Function: Deriving Biologically Relevant Functions from Statistically Significant Functions. Briefings in Bioinformatics, 13, 216-227. https://doi.org/10.1093/bib/bbr041

[15] Wang, H., Xie, Z., Hou, T., Li, Z., Huang, K., Gong, J., et al. (2017) MiR-125b Regulates the Osteogenic Differentiation of Human Mesenchymal Stem Cells by Targeting BMPR1b. Cellular Physiology and Biochemistry, 41, 530-542.

https://doi.org/10.1159/000457013

[16] Xu, S., Cecilia Santini, G., De Veirman, K., VandeBroek, I., Leleu, X., De Becker, A., et al. (2013) Upregulation of miR-135b Is Involved in the Impaired Osteogenic Differentiation of Mesenchymal Stem Cells Derived from Multiple Myeloma Patients. PLOS ONE, 6, e79752. https://doi.org/10.1371/journal.pone.0079752

[17] Sato, M.M., Nashimoto, M., Katagiri, T., Yawaka, Y. and Tamura, M. (2009) Bone Morphogenetic Protein-2 Down-Regulates miR-206 Expression by Blocking Its Maturation Process. Biochemical and Biophysical Research Communications, 22, 125-129. https://doi.org/10.1016/j.bbrc.2009.03.142

[18] Wang, X., Guo, B., Li, Q., Peng, J., Yang, Z., Wang, A., et al. (2013) MiR-214 Targets ATF4 to Inhibit Bone Formation. Nature Medicine, 19, 93-100. https://doi.org/10.1038/nm.3026

[19] Hupkes, M., Sotoca, A.M., Hendriks, J.M., van Zoelen, E.J. and Dechering, K.J. (2014) MicroRNA miR-378 Promotes BMP2-Induced Osteogenic Differentiation of Mesenchymal Progenitor Cells. BMC Molecular Biology, 27, 15.

[20] Guo, D., Li, Q., Lv, Q., Wei, Q., Cao, S. and Gu, J. (2014) MiR-27a Targets sFRP1 in hFOB Cells to Regulate Proliferation, Apoptosis and Differentiation. PLoS ONE, 13, e91354. https://doi.org/10.1371/journal.pone.0091354

[21] Hu, R., Liu, W., Li, H., Yang, L., Chen, C., Xia, Z.Y., et al. (2011) A Runx2/miR-3960/miR-2861 Regulatory Feedback Loop during Mouse Osteoblast Differentiation. The Journal of Biological Chemistry, 8, 12328-12339. https://doi.org/10.1074/jbc.M110.176099

[22] Iwawaki, Y., Mizusawa, N., Iwata, T., Higaki, N., Goto, T., Watanabe, M., et al. (2015) MiR-494-3p Induced by Compressive Force Inhibits Cell Proliferation in 
MC3T3-E1 Cells. Journal of Bioscience and Bioengineering, 120, 456-462. https://doi.org/10.1016/j.jbiosc.2015.02.006

[23] Zhi, X., Wu, K., Yu, D., Wang, Y., Yu, Y., Yan, P. and Lv, G. (2016) MicroRNA-494 Inhibits Proliferation and Metastasis of Osteosarcoma through Repressing Insulinreceptor Substrate-1. American Journal of Translational Research, 15, 3439-3447.

[24] Weng, J.H., Yu, C.C., Lee, Y.C., Lin, C.W., Chang, W.W. and Kuo, Y.L. (2016) mMiR-494-3p Induces Cellular Senescence and Enhances Radiosensitivity in $\mathrm{Hu}-$ man Oral Squamous Carcinoma Cells. International Journal of Molecular Sciences, 17, 1092.

[25] Colden, M., Dar, A.A., Saini, S., Dahiya, P.V., Shahryari, V., Yamamura, S., et al. (2017) MicroRNA-466 Inhibits Tumor Growth and Bone Metastasis in Prostate Cancer by Direct Regulation of Osteogenic Transcription Factor RUNX2. Cell Death \& Disease, 26, e2572. https://doi.org/10.1038/cddis.2017.15

[26] Seo, M., Choi, J.S., Rho, C.R., Joo, C.K. and Lee, S.K. (2015) MicroRNA miR-466 Inhibits Lymphangiogenesis by Targeting Prospero-Related Homeobox 1 in the $\mathrm{Al}$ kali Burn Corneal Injury Model. Journal of Biomedical Science, 22, 3. https://doi.org/10.1186/s12929-014-0104-0

[27] Min, Z., Zhang, R., Yao, J., Jiang, C., Guo, Y., Cong, F., et al. (2015) MicroRNAs Associated with Osteoarthritis Differently Expressed in Bone Matrix Gelatin (BMG) Rat Model. International Journal of Clinical and Experimental Medicine, 15, 1009-1017.

[28] Zhao, Y., Yan, M., Yun, Y., Zhang, J., Zhang, R., Li, Y., et al. (2017) MicroRNA-455-3p Functions as a Tumor Suppressor by Targeting eIF4E in Prostate Cancer. Oncology Reports, 37, 2449-2458. https://doi.org/10.3892/or.2017.5502

[29] Hao, C., Yang, S., Xu, W., Shen, J.K., Ye, S., Liu, X., et al. (2016) MiR-708 Promotes Steroidinduced Osteonecrosis of Femoral Head, Suppresses Osteogenic Differentiation by Targeting SMAD3. Scientific Reports, 2, Article No. 22599. https://doi.org/10.1038/srep22599

[30] Pourshafie, N., Lee, P.R., Chen, K.L., Harmison, G.G., Bott, L.C., Katsuno, M., et al. (2016) MiR-298 Counteracts Mutant Androgen Receptor Toxicity in Spinal and Bulbar Muscular Atrophy. Molecular Therapy, 24, 937-945. https://doi.org/10.1038/mt.2016.13

[31] Barbagallo, D., Piro, S., Condorelli, A.G., Mascali, L.G., Urbano, F., Parrinello, N., et al. (2013) MiR-296-3p, MiR-298-5p and Their Downstream Networks Are Causally Involved in the Higher Resistance of Mammalian Pancreatic $\alpha$ Cells to Cytokine-Induced Apoptosis as Compared to $\beta$ Cells. BMC Genomics, 14, 62. https://doi.org/10.1186/1471-2164-14-62

[32] Jian, X., Li, X., Wu, F., Gao, H., Wang, G., Zheng, H., et al. (2017) Overexpression of miR-92a Promotes the Tumor Growth of Osteosarcoma by Suppressing F-boxandWD Repeat-Containing Protein 7. Gene, 606, 10-16.

https://doi.org/10.1016/j.gene.2017.01.002

[33] Penzkofer, D., Bonauer, A., Fischer, A., Tups, A., Brandes, R.P., Zeiher, A.M., et al. (2014) Phenotypic Characterization of miR-92a-/-mice Reveals an Important Function of miR-92a Inskeletal Development. PLOS ONE, 30, e101153. https://doi.org/10.1371/journal.pone.0101153

[34] Wang, Z., Lu, Y., Zhang, X., Ren, X., Wang, Y., Li, Z., et al. (2012) Serum Microrna Is a Promising Biomarker for Osteogenesis Imperfecta. Intractable \& Rare Diseases Research, 1, 81-85.

[35] Geng, S., Gu, L., Ju, F., Zhang, H., Wang, Y., Tang, H., et al. (2016) MicroRNA-224 
Promotes the Sensitivity of Osteosarcoma Cells to Cisplatin by Targeting Rac1. Journal of Cellular and Molecular Medicine, 20, 1611-1619. https://doi.org/10.1111/jcmm.12852

[36] Huang, L., Dai, T., Lin, X., Zhao, X., Chen, X., Wang, C., et al. (2012) MicroRNA-224 Targets RKIP to Control Cell Invasion and Expression of Metastasis Genes in Human Breast Cancer Cells. Biochemical and Biophysical Research Communications, 24, 127-133. https://doi.org/10.1016/j.bbrc.2012.07.025

[37] Gennari, L., Bianciardi, S. and Merlotti, D. (2017) MicroRNAs in Bone Diseases. Osteoporosis International, 28, 1191-1213.

https://doi.org/10.1007/s00198-016-3847-5 